\title{
ELABORACIÓN DE JAMÓN COCIDO DE PATO CRIOLLO (CAIRINA MOSCHATA) BAJO EN GRASA
}

\section{DEVELOPMENT OF DUCK COOKED HAM (CAIRINA MOSCHATA) LOW FAT}

\footnotetext{
Universidad Católica de Santiago de Guayaquil.jorge.velasquez02@cu.ucsg.edu.ec

2 Universidad de La Habana (Cuba). mrocaa@iiia.edu.cu

3 Universidad de Guayaquil. raul.diazt@ug.edu.ec
}

\begin{abstract}
RESUMEN
Se elaboraron 16 formulaciones de jamón cocido de pato, las que fueron definidas a partir del empleo del programa Design Expert, utilizando restricciones para los contenidos de carne, antioxidantes y harina de subproductos de palmito. Durante el proceso de optimización quedó demostrado que la incorporación de harina de subproductos de palmito solo es posible a niveles inferiores al $0,5 \%$, lo que significa un incremento poco significativo del contenido de fibra y que los niveles de antioxidantes no deben exceder de 0,1\%. A partir de estos resultados, se elaboraron varias fórmulas de jamón cocido de pato conteniendo salvado de arroz. Se demostró que es posible elaborar fórmulas de jamón a base de carne de pato, con un 3\% de salvado de arroz, con características físicoquímicas y sanitarias que cumplen con la legislación vigente, que poseen características sensoriales aceptables por los consumidores y que pueden ser comercializadas hasta al menos 28 días después de su elaboración.
\end{abstract}

PALABRAS ClaVE: jamón cocido de pato, salvado de arroz, harina de subproductos de palmito, fibra.
ABSTRACT 16 formulations duck ham, which were defined from the use of Design Expert program using restrictions on the contents of meat, antioxidants and palm by-products flour. During the optimization process demonstrated that incorporating palm by-products flour is only possible levels below $0.5 \%$, which means an insignificant increase of fiber content and antioxidant levels should not exceed $0.1 \%$. From these results, several duck's ham formulas with rice bran were developed. It proved to be possible to develop formulas ham meat duck, with $3 \%$ rice bran, chemical and physical health characteristics that complies with current legislation, which possess acceptable sensory characteristics by consumers and can be comercialized until at least 28 days after processing.

KEYWORDS: cooked ham, rice bran, palm by-products flour, fiber. 
INTRODUCCIÓN

La carne y los productos cárnicos son una parte esencial de las dietas y aportan un gran número de nutrientes, siendo la fuente fundamental de proteínas de alto valor biológico. Además son una buena fuente de hierro, zinc y fósforo, con cantidades significativas de otros elementos traza como el selenio, magnesio y cobalto, y representa una excelente fuente de vitaminas del grupo B, por lo que ha sido tradicionalmente muy apreciada (Jiménez, Sánchez y Olmedilla, 2011). Los principales problemas de salud asociados a los productos cárnicos son el nivel de sodio y grasa, y en productos curados, el nivel de nitritos utilizados. Por esto, empleando otros ingredientes como hidrocoloides o gomas en la formulación se puede lograr una reducción considerable en los niveles de grasa (Totosaus, 2007).

Debido a que los problemas de salud, como la obesidad, las enfermedades del corazón y la diabetes, han aumentado en muchos países del mundo, la industria de alimentos se ha enfrentado a una creciente presión para mejorar la calidad nutricional de sus productos (Ospina, Restrepo, y López, 2011). Dentro de estas nuevas tendencias se ha suscitado un gran interés, especialmente dentro del sector cárnico, hacia el desarrollo de productos bajos en grasa, debido en gran parte a la alta incidencia que ha venido teniendo el consumo de estos productos en el desarrollo de ciertas enfermedades por los altos contenidos de grasa que suelen tener, especialmente grasa animal (Pacheco, Restrepo y Sepúlveda, 2011).

Una atención particular se ha dirigido a los problemas de salud asociados con el contenido de grasa en los alimentos. Los consumidores buscan productos cárnicos bajos en grasa o sin ella (Ospina, Restrepo y López, 2011). Los mayores componentes de la grasa dietética son los triglicéridos, los cuales varían en los ácidos grasos que contienen y/o en la implantación estereoquímica de los diferentes ácidos grasos en las tres posiciones del glicerol. El tipo de grasa consumida es un factor importante que contribuye al desarrollo de enfermedades cardiovasculares, cáncer, diabetes, y otros tipos de enfermedades degenerativas. Sin embargo, las grasas o lípidos son fuente de energía y proveen sustancias que regulan procesos fisiológicos importantes (Totosaus, 2011).

Normalmente la grasa en embutidos se utiliza de un 15 al 20\% (Totosaus, 2007). Sin embargo, con el exceso de la reducción de grasa, los productos pueden perder palatabilidad, volverse secos, y la textura puede ser más dura, lo que resulta en productos menos aceptables para los consumidores (Ali, Kim, Seo, Jung, Kim, Yang y Joo, 2011).

Además, la calidad de estos productos cambia según el tratamiento tecnológico y durante su almacenamiento, como se demostró en una investigación realizada con el jamón de cerdo (González, Suárez y Martínez, 2009).

Es de señalar que en una investigación donde se elaboraron salchichones enriquecidos con calcio y reducidos en grasa, la mayor valoración sensorial se obtuvo en los lotes elaborados con menor contenido graso (Soto, García y Selgas, 2009), lo que demuestra la factibilidad de producir este tipo de alimentos.

Por estos motivos se han empleado diferentes materiales como sustitutos de la grasa animal en la elaboración de productos cárnicos, entre ellos aceites vegetales como el de soya y girasol (Rey y Gualdron, 2011), mezclas de aceite de oliva y margarina (Ninco, Cuasquer, Bonilla, Carrero, y Rey, 2010), así como el aceite de oliva, proteínas de soya y antioxidantes como el romero y la salvia, y fibra (Ospina, Restrepo y López, 2011).

Algunas especias antioxidantes como el romero y otras, han demostrado afectar al color de los productos cárnicos, incluidos los de carne de aves. Sin embargo, en algunos de estos productos, un aumento en el color rojo puede ser deseado. En todo caso, al seleccionar un antioxidante natural, el impacto sensorial y en la calidad del producto debe ser considerada para el logro de las características deseadas (Karre, López \& Getty, 2013).

En cuanto a la fibra dietética, su papel ha sido bien documentado en el control de diversas enfermedades crónicas como el cáncer de colon, la diverticulitis, y las enfermedades cardiovasculares, entre otras. La fibra dietética puede ser añadida a los productos cárnicos a través de varias fuentes como vegetales, frutas, leguminosas, cereales, etc. en forma de extensores, rellenos u otros. Además de añadir un ingrediente importante a los productos cárnicos se mejoran otras características de los productos y se incrementan las ganancias marginales de la industria (Bhat y Bhat, 2011).

El objetivo de esta investigación fue determinar cómo influyen la adición de fuentes de fibra dietética y el uso de antioxidantes naturales sobre las características de calidad del jamón de pato, de bajo contenido graso. 
METODOLOGÍA

La elaboración del jamón se concibió en dos etapas. En la primera, se realizó un diseño experimental utilizando el programa de optimización Design Expert, con las siguientes restricciones: carne de pato en el rango entre 54 y 63,5\%, harina de subproductos de palmito entre 0 y $9 \%$ y la mezcla de antioxidantes entre 0 y $0,5 \%$. El programa de optimización recomendó realizar 16 formulaciones, incluidas las repeticiones para evaluar la varianza del error puro.

TABLA 1. FORMULACIONES DEL EXPERIMENTO

\begin{tabular}{llll}
\hline \multirow{2}{*}{ TIPO DE VENTA } & \multicolumn{3}{c|}{ FRECUENCIA PORCENTAJE } \\
\cline { 2 - 4 } & Carne de pato & $\begin{array}{l}\text { Harina de subproductos } \\
\text { de Palmito }\end{array}$ & $\begin{array}{l}\text { Anti } \\
\text { oxidantes }\end{array}$ \\
\hline 1 & 57 & 6 & 0,5 \\
\hline 2 & 61 & 2 & 0,25 \\
\hline 3 & 63,5 & 0 & 0 \\
\hline 4 & 59 & 5 & 0 \\
\hline 5 & 54 & 9 & 0,5 \\
\hline 6 & 54,5 & 9 & 0 \\
\hline 7 & 60 & 3 & 0,5 \\
\hline 8 & 54,25 & 9 & 0,25 \\
\hline 9 & 54 & 9 & 0,5 \\
10 & 63 & 0 & 0,5 \\
\hline 11 & 54,5 & 9 & 0 \\
\hline 12 & 57 & 7 & 0,125 \\
\hline 13 & 63,5 & 0 & 0 \\
\hline 14 & 54,25 & 9 & 0,25 \\
\hline 15 & 63 & 0 & 0,5 \\
\hline 16 & 60,5 & 3 & 0 \\
\hline
\end{tabular}

Fuente: los autores.

En la segunda etapa, basados en los resultados obtenidos, se realizaron nuevas formulaciones (tabla 2) incorporando salvado de arroz como sustituto de la harina de soya o la fécula de maíz, en lugar de la harina de subproductos de palmito.

TABLA 2. REDISEÑO DEL PRODUCTO

\begin{tabular}{lllll} 
FórmuLA & $\begin{array}{l}\text { CARNE DE } \\
\text { PATO (\%) }\end{array}$ & $\begin{array}{l}\text { HARINA DE } \\
\text { SOYA (\%) }\end{array}$ & $\begin{array}{l}\text { SALVADO DE } \\
\text { ARROZ (\%) }\end{array}$ & $\begin{array}{l}\text { FÉCULA DE } \\
\text { MAÍz (\%) }\end{array}$ \\
\hline 1 & 63 & 3 & 0 & 3 \\
2 & 63 & 2 & 1 & 3 \\
3 & 63 & 1 & 2 & 3 \\
\hline 4 & 63 & 0 & 3 & 3 \\
\hline 5 & 63 & 0 & 6 & 0 \\
\hline
\end{tabular}

Fuente: los autores.

A las formulaciones propuestas se les realizaron los siguientes análisis en tiempo cero:

- Químicos: humedad (método gravimétrico, secado en estufa a $105^{\circ} \mathrm{C}$ hasta peso constante), proteína por método microkjeldalh ba- sado en una digestión ácida (método AOAC 954.01), grasa (método Folch Modificado), fibra cruda total (Método AOAC 978.10), pH (método potenciométrico) y acidez (por valoración, expresada como ácido láctico).

- Microbiológicos: recuento total de microorganismos aerobios mesófilos (recuento en placa), determinación de Escherichia coli, Salmonella y Staphylococcus aureus (NTE INEN 1 338:2010).

- Sensoriales: prueba de aceptación-rechazo por atributo (aspecto, olor, sabor y textura) en la primera etapa y prueba de preferencia en la segunda realizada por 98 consumidores potenciales del producto.

De acuerdo a los resultados obtenidos, se seleccionaron las dos formulaciones preferidas para evaluar el comportamiento durante el almacenamiento a $4{ }^{\circ} \mathrm{C}$, por un período de 28 días.

Durante el almacenamiento de las muestras se evaluaron, cada siete días, su humedad, $\mathrm{pH}$ y acidez, recuento total de microorganismos aerobios mesófilos (recuento en placa), determinación de Escherichia coli, Salmonella y Staphylococcus aureus. De acuerdo a los métodos antes citados, se realizó una prueba descriptiva de perfil, empleando 9 jueces entrenados utilizando la escala mostrada en la ficha de evaluación de las características descriptivas del jamón de pato.

RESULTADOS

Los resultados de las pruebas fisicoquímicas realizadas a las 16 formulaciones estudiadas se muestran en la tabla 3.

TABLA 3. RESULTADOS DE LOS ANÁLISIS FÍSICO-QUÍMICOS DE LAS 16 FORMULACIONES ESTUDIADAS (MEDIA DE 2 DETERMINACIONES) trataMiento huMEdad proteína grasa fibra acidez PH

\begin{tabular}{lllllll}
1 & 70,96 & 17,21 & 7,03 & 0,75 & 0,88 & 6,5 \\
\hline 2 & 73,17 & 16,21 & 4,89 & 0,52 & 0,90 & 6,4 \\
3 & 75,02 & 16,87 & 5,05 & 0,86 & 0,89 & 6,4 \\
\hline 4 & 71,63 & 17,14 & 4,88 & 0,58 & 0,87 & 6,6 \\
5 & 68,93 & 16,57 & 4,79 & 0,82 & 0,87 & 6,6 \\
6 & 68,94 & 16,12 & 4,95 & 0,73 & 0,87 & 6,6 \\
7 & 72,99 & 17,04 & 5,16 & 0,69 & 0,91 & 6,4 \\
8 & 68,94 & 15,64 & 5,65 & 0,82 & 0,88 & 6,6 \\
9 & 69,03 & 17,29 & 5,51 & 0,79 & 0,87 & 6,6 \\
10 & 74,98 & 16,82 & 4,58 & 0,70 & 0,89 & 6,4 \\
11 & 68,94 & 16,72 & 5,82 & 0,79 & 0,89 & 6,4 \\
12 & 70,29 & 16,67 & 5,09 & 0,91 & 0,87 & 6,6 \\
13 & 75,01 & 17,13 & 5,01 & 0,42 & 0,90 & 6,4 \\
14 & 68,94 & 17,17 & 6,63 & 0,91 & 0,92 & 6,5 \\
15 & 75,03 & 17,09 & 5,23 & 0,78 & 0,89 & 6,5 \\
16 & 73,09 & 16,29 & 5,46 & 0,54 & 0,89 & 6,4 \\
\hline
\end{tabular}

Fuente: los autores. 
Estos valores fueron ingresados al programa de optimización Design Expert, obteniéndose los resultados que se muestran en la tabla 4. Los resultados microbiológicos no fueron contemplados en el proceso de optimización, pues todas las formulaciones cumplieron con las especificaciones de la NTE INEN 1 338:2010. El índice de costo depende de la formulación seleccionada y no de la tecnología empleada, pues en este caso se utilizó la misma tecnología para todas las formulaciones. Por este motivo, tampoco fue contemplado este indicador en la optimización.

\section{TABLA 4. RESULTADOS DEL PROCESO DE OPTIMIZACIÓN}

\begin{tabular}{|rrrrr|}
\hline Solución & \multicolumn{3}{c}{$\begin{array}{c}\text { PORCENTAJES } \\
\text { Harina de }\end{array}$} & \multicolumn{1}{c|}{ AJUSTES } \\
\cline { 2 - 5 } & Carne de pato & $\begin{array}{r}\text { Habproductos } \\
\text { de palmito }\end{array}$ & Anti oxidante & Deseabilidad \\
\hline 1 & 62.970 & 0.213 & 0.317 & $0.956^{* *}$ \\
\hline 2 & 63.319 & 0.181 & 0.000 & 0.941 \\
\hline 3 & 57.336 & 5.780 & 0.384 & 0.244 \\
\hline 4 & 54.503 & 8.997 & 0.000 & 0.020 \\
\hline 5 & 54.249 & 9.000 & 0.251 & 0.004 \\
\hline
\end{tabular}

Fuente: los autores.

Dado que la fórmula propuesta por el programa presenta un porcentaje muy bajo de harina de subproductos de palmito, el objetivo de incorporar fibra a partir de este componente no se alcanza, por lo que se pasó a un rediseño del producto. Las fórmulas empleadas fueron sometidas a una prueba de ordenamiento por preferencia, obteniéndose los resultados mostrados en la tabla 5.

\section{TABLA 5. RESULTADOS DE LA PRUEBA DE ORDENAMIENTO}

\begin{tabular}{|c|c|c|c|c|c|c|}
\hline \multirow{2}{*}{ FORMULA } & \multicolumn{5}{|c|}{ POSICIÓN OTORGADA } & \multirow{2}{*}{ SUMATORIA } \\
\hline & 1 & 2 & 3 & 4 & 5 & \\
\hline 1 & 10 & 16 & 14 & 26 & 32 & 328 \\
\hline 2 & 18 & 18 & 20 & 32 & 12 & 302 \\
\hline 3 & 16 & 26 & 18 & 22 & 14 & 280 \\
\hline 4 & 36 & 14 & 32 & 8 & 8 & 232 \\
\hline 5 & 18 & 24 & 14 & 10 & 32 & 308 \\
\hline $\begin{array}{l}\text { Total de } \\
\text { jueces }\end{array}$ & 98 & 98 & 98 & 98 & 98 & \\
\hline
\end{tabular}

Fuente: Los autores.

Los resultados de los análisis fisicoquímicos realizados a las cinco formulaciones se muestran en la tabla 6 mientras que los resultados de los análisis microbiológicos se muestran en la tabla 8 .
TABLA 6. RESULTADOS DE LOS ANÁLISIS FÍSICO-QUÍMICOS DE LAS FÓRMULAS CON SALVADO DE ARROZ (MEDIA DE 2 DETERMINACIONES)

\begin{tabular}{lrrrrr} 
FóRMULA & $\begin{array}{r}\text { Proteína } \\
\mathrm{g} / 100 \mathrm{~g}\end{array}$ & $\begin{array}{r}\text { Lípidos } \\
\text { Totales } \\
\mathrm{g} / 100 \mathrm{~g}\end{array}$ & $\begin{array}{r}\text { Humedad } \\
\mathrm{g} / 100 \mathrm{~g}\end{array}$ & $\begin{array}{r}\text { Cenizas } \\
\mathrm{g} / 100 \mathrm{~g}\end{array}$ & $\begin{array}{r}\text { Fhbra } \\
\mathrm{g} / 100 \mathrm{~g}\end{array}$ \\
\hline 1 & 16.85 & 3.97 & 75.36 & 3.5 & 2.41 \\
\hline 2 & 15.26 & 4.14 & 73.97 & 3.77 & 3.57 \\
\hline 3 & 16.28 & 4.86 & 75.6 & 3.67 & 3.35 \\
\hline 4 & 14.29 & 5.72 & 72.19 & 3.99 & 3.43 \\
\hline
\end{tabular}

Fuente: los autores.

Las fórmulas seleccionadas ( 1 y 4) fueron sometidas a un estudio de almacenamiento a $4^{\circ} \mathrm{C}$ por 28 días. Las tablas 6 y 7 muestran los resultados de los análisis efectuados durante el almacenamiento de ambas fórmulas.

TABLA 7. RESULTAdos dE LOS ANÁLISIS FísICO-QUíMICOS REALIZADOS DURANTE EL ALMACENAMIENTO REFRIGERADO (MEDIA DE 2 DETERMINACIONES)

\begin{tabular}{rrrrr} 
FórMula & Tiempo (días) & Humedad & Acidez & pH \\
\hline & 0 & 73,98 & 0,61 & 6,01 \\
\hline 1 & 72,80 & 0,65 & 6,04 \\
\hline 14 & 73,27 & 0,71 & 6,09 \\
\hline 21 & 73,70 & 0,59 & 6,22 \\
\hline 28 & 75,00 & 0,68 & 6,18 \\
\hline & 0 & 73,37 & 0,76 & 5,93 \\
\hline & 7 & 73,17 & 0,85 & 6,02 \\
& 14 & 71,86 & 0,74 & 6,22 \\
& 21 & 73,24 & 0,55 & 6,22 \\
\hline 28 & 73,22 & 0,61 & 6,18 \\
\hline
\end{tabular}

Fuente: los autores.

TABLA 8. RESULTADOS DE LOS ANÁLISIS MICROBIOLÓGICOS REALIZADOS DURANTE EL ALMACENAMIENTO REFRIGERADO (MEDIA DE 2 DETERMINACIONES)

\begin{tabular}{rrrrrr} 
FóRMULA & $\begin{array}{r}\text { Tiempo } \\
\text { (días) }\end{array}$ & $\begin{array}{r}\text { Aerohios } \\
\text { Mesofilos } \\
\text { (UFC/g) }\end{array}$ & $\begin{array}{r}\text { E. Coli } \\
\text { (UFC/g) }\end{array}$ & $\begin{array}{r}\text { Salmonella } \\
\text { (UFC/g) }\end{array}$ & $\begin{array}{r}\text { Staphylococcus } \\
\text { (UFC/g) }\end{array}$ \\
\hline 0 & $120 \times 10^{2}$ & $<10$ & Ausencia & $<10$ \\
1 & 7 & $<100$ & $<10$ & Ausencia & $<10$ \\
& 14 & $26 \times 10^{2}$ & $<10$ & Ausencia & $<10$ \\
\hline 21 & $7 \times 10^{3}$ & $<10$ & Ausencia & $<10$ \\
28 & $18 \times 10^{3}$ & $<10$ & Ausencia & $<10$ \\
& 0 & $<100$ & $<10$ & Ausencia & $<10$ \\
& 7 & $28 \times 10^{2}$ & $<10$ & Ausencia & $<10$ \\
4 & 14 & 10 & $<10$ & Ausencia & $<10$ \\
& 21 & $15 \times 10^{3}$ & $<10$ & Ausencia & $<10$ \\
28 & $16 \times 10^{3}$ & $<10$ & Ausencia & $<10$ \\
\hline
\end{tabular}

Fuente: los autores. 


\section{DISCUSIÓN DE RESULTADOS}

Como puede apreciarse en la tabla 4, solo dos de las formulaciones presentan un alto grado de deseabilidad, es decir se ajustan en más de un 94\% a las restricciones impuestas. Estas dos fórmulas (1 y 2) presentan niveles de adición de harina de subproductos de palmito extremadamente bajos, lo que nos indica que este subproducto no es adecuado para la elaboración de este tipo de jamón. Además, tanto los resultados de la optimización como los comentarios de los jueces, indicaron la necesidad de reducir el porcentaje de antioxidante, debido a que le confiere un sabor no característico al producto.

Al realizar la reformulación del producto, se desarrolló una prueba de preferencia para los jamones elaborados con el salvado de arroz (tabla 5). Para una prueba de preferencia, la tabla de diferencia de sumatoria ordinal absoluta crítica de todos los tratamientos con un nivel de significancia del 5\%, nos indica que para 5 muestras y 100 juicios, serán diferentes aquellos tratamientos cuya diferencia sea igual o mayor a 61 (Espinosa, 2007).

Sólo la fórmula 4 difiere significativamente del patrón, por lo que esta conformará, junto al patrón, las fórmulas seleccionadas para las pruebas de almacenamiento. Como puede observarse en las tablas 7 y 8 , ambas fórmulas fueron estables durante el tiempo de almacenamiento, cumpliendo con la norma ecuatoriana NTE INEN 1 339:96 que indica que el $\mathrm{pH}$ del jamón cocido debe estar entre 5,8 y 6,2 y que el recuento total en placa de microorganismos mesófilos aerobios no debe exceder de 5,0 x $10^{5}$. Las unidades formadoras de colonia/g para Staphylococcus aureus no deben sobrepasar $1 \times 10^{3}$, las unidades formadoras de colonia/g para Escherichia coli deben ser menores que 3 , y la Salmonella debe estar ausente, cumpliendo con la norma ecuatoriana NTE INEN 1338:2010. Por tanto, la adición del salvado de arroz no afecta la calidad sanitaria del producto elaborado, además, estos resultados indican que tanto el proceso de elaboración como el tratamiento térmico fueron adecuados.

Las dos fórmulas fueron caracterizadas sensorialmente por un grupo de jueces adiestrados. El resultado de esta prueba se muestra en la tabla 9.

La incorporación del salvado de arroz prácticamente no afecta la caracterización sensorial del producto. Debe señalarse que de acuerdo a la escala desarrollada por los jueces, en los
TABLA 9. CARACTERIZACIÓN SENSORIAL DE LAS FÓRMULAS SELECCIONADAS

\begin{tabular}{|c|c|c|c|}
\hline FÓRMULA & INDICADOR & FÓRMULA 1 & FÓRMULA 4 \\
\hline \multirow{5}{*}{$\begin{array}{l}\text { Aspecto al } \\
\text { corte }\end{array}$} & Porosidad & Ligeramente poroso & Ligeramente poroso \\
\hline & Marmoleado & $\begin{array}{l}\text { Ligeramente } \\
\text { marmoleado }\end{array}$ & $\begin{array}{l}\text { Ligeramente } \\
\text { marmoleado }\end{array}$ \\
\hline & Color rosado & Ligero & Ligero \\
\hline & Brillo & Ausente a ligero & Ausente a ligero \\
\hline & Humedad & $\begin{array}{l}\text { Ligeramente } \\
\text { húmedo }\end{array}$ & Ligeramente húmedo \\
\hline \multirow{4}{*}{ Olor } & Ahumado & Ligero & Ligero \\
\hline & Olor graso & Ausente a ligero & Ausente a ligero \\
\hline & Olor rancio & Ausente a ligero & Ausente a ligero \\
\hline & Olor ácido & Ligero & Ligero \\
\hline \multirow{3}{*}{ Sabor } & Sabor salado & $\begin{array}{l}\text { Ligero a Caracte- } \\
\text { rístico }\end{array}$ & Característico \\
\hline & Sabor rancio & Ausente a ligero & Ausente a ligero \\
\hline & Sabor ácido & Ausente a ligero & Ausente a ligero \\
\hline \multirow{5}{*}{ Textura } & Dureza & Característica & $\begin{array}{l}\text { Ligera a } \\
\text { Característica }\end{array}$ \\
\hline & S. grasa & Ausente a Ligera & Ligera \\
\hline & $\mathrm{S}$, húmeda & Ligera & Ligera \\
\hline & Arenosidad & Ligera & Ligera \\
\hline & Masticabilidad & Ligera & Ligera \\
\hline
\end{tabular}

Fuente: los autores.

atributos relativos al olor, sabor y textura, un indicador con la calificación Ligero es menos intenso que Característico, por lo que las notas atribuidas a estos indicadores pueden considerarse positivas en sentido general.

Las figuras 1 y 2 muestran el comportamiento de las dos fórmulas durante el almacenamiento.

Uno de los defectos que puede aparecer en este tipo de jamón, es el incremento del sabor rancio, lo que no ocurrió en este producto, indicando que el porcentaje de antioxidante empleado es efectivo. Como las características fisicoquímicas, microbiológicas y sensoriales no se alteran en el tiempo estudiado, se puede asegurar que es posible elaborar fórmulas de jamón empacado a base de carne de pato, en funda sintética, con características fisicoquímicas y sanitarias que cumplen con la legislación vigente, y características sensoriales aceptables por los consumidores, para su comercializarse dentro de los 28 días posteriores a su elaboración.

Se requerirán estudios posteriores de almacenamiento del mismo jamón, pero empacado al vacío, donde se supone existe un incremento de la vida útil, en al menos, el doble de tiempo. 


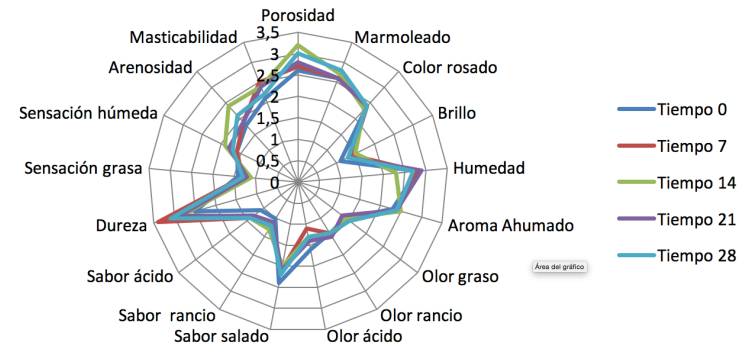

Figura 1. Análisis de perfil de la fórmula 1 Fuente: los autores.

\section{REFERENCIAS. BIBLIOGRÁFICAS}

Ali, M. S., Kim, G. D., Seo, H. W., Jung, E. Y., Kim, B. W., Yang, H. S., y Joo, S. T. (2011). Possibility of Making Low-fat Sausages from Duck Meat with Addition of Rice Flour. Asian - Australia Journal of Animal Science, 24 (03), 421 - 428. Recuperado de http://www.koreascience.or.kr/article/ArticleFullRecord.jsp?cn=E1DMBP_2011_v24n3_421

Bhat, Z.F., Bhat, H. (2011). Fibre-based functional meat products. Asian Journal of Food and Agro-Industry 04 (04), 261 - 273. Recuperado de http://www.ajofai.info/Abstract/Fibrebased $\% 20$ functional $\% 20$ meat $\% 20$ products.pdf

Espinosa, J. (2007). Evaluación sensorial de los alimentos. Editorial Universitaria. La Habana, 18-30.

González, M.I., Suárez, H. y Martínez, O.L. (2009). Relación entre las características fisicoquímicas y sensoriales en jamón de cerdo durante el proceso de cocción y temperatura de almacenamiento. Vitae, Revista de la Facultad de Química Farmacéutica 16 (02), 183 - 189. Recuperado de http://www. scielo.org.co/scielo.php?script=sci_arttext\&pi $\mathrm{d}=$ S0121-40042009000200002

Jiménez, F., Sánchez, F.J., y Olmedilla, B. (2010). Design and development of meat-based functional foods with walnut: Technological, nutritional and health impact. Food Chemistry, 123 (04), 959 - 967. Recuperado de http://www.sciencedirect.com/ science/article/pii/S0308814610006783

Karre, L., Lopez, K., y Getty, K.J.K. (2013). Natural antioxidants in meat and poultry products. Meat Science 94 (02), 220 - 227. Recuperado de http:// www.sciencedirect.com/science/article/pii/ S0309174013000302

Ninco, A.R., Cuasquer, L.P., Bonilla, S.M., Carrero, M.B. y Rey J.F. (2010). Evaluación de un producto cárnico de caprino con sustitución de grasa. Épsilon, 14, 59 - 87. Recuperado de http://revistas.lasalle.edu.co/index.php/ep/article/view/897/805

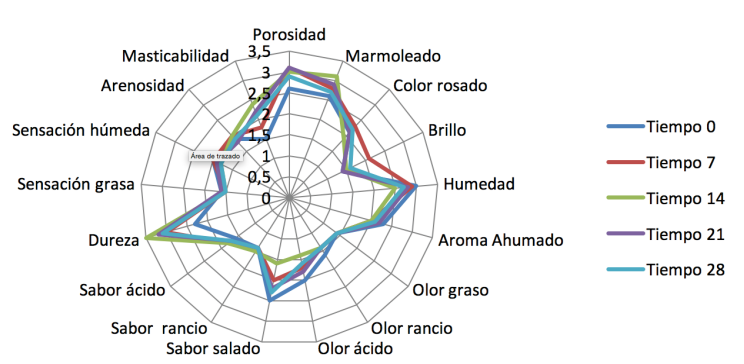

Figura 2. Análisis de perfil de la fórmula 4 Fuente: los autores.

Ospina, S.M., Restrepo D.A., y López, JH. (2011). Derivados cárnicos como alimentos funcionales. Revista Lasallista de Investigación, 08 (02), 163 - 172. Recuperado de http://www.scielo.org. co/pdf/rlsi/v8n2/v8n2a18.pdf

Pacheco, W.A., Restrepo, D.A. y Sepúlveda, J.U. (2011). Revisión: Uso de Ingredientes no Cárnicos como Reemplazantes de Grasa en Derivados Cárnicos Revista Facultad Nacional de Agronomía Medellín 64 (2). Recuperado de http://www.scielo.org.co/ $\mathrm{pdf} / \mathrm{rfnam} / \mathrm{v} 64 \mathrm{n} 2 / \mathrm{v} 64 \mathrm{n} 2 \mathrm{a} 23 . \mathrm{pdf}$

Rey, J.F. y Gualdron, L. (2011). Evaluación de la Sustitución de Grasa Animal por Grasa Vegetal Insaturada en la Elaboración de un Embutido de Carne de Búfalo (Bubalus bubalis). Información Tecnológica, 22 (02), 43 - 54. Recuperado de http://www.scielo.cl/scielo.php?pid=S071807642011000200006\&script=sci_arttext

Soto, A.M., García, M.L y Selgas, M.D. (2009). Productos Cárnicos Madurados Enriquecidos Con Gluconato D-Cálcico. Revista Complutense de Ciencias, 3 (02) 41 - 50. Recuperado de http:// revistas.ucm.es/index.php/RCCV/article/view/ RCCV0909220041A/22362

Totosaus, A. (2007). Productos cárnicos emulsionados bajos en grasa y sodio. Nacameh 1 (01), $53-66$. Recuperado de https://scholar.google.com.ec/sc holar?q=Nacameh $+01+\% 2801 \% 29 \% 2 \mathrm{C}+53+\% \mathrm{E} 2 \% 80$ $\% 93+66 \& b t n G=\& h l=e s \& a s \_s d t=0 \% 2 C 5$

Totosaus, A. (2011). Aceites y grasas vegetales como ingrediente funcional en productos cárnicos. Nacameh 5 (01), S108 - S118. Recuperado de https:// scholar.google.com.ec/scholar?cluster=159617 36960140672942\&hl=es\&as_sdt $=0,5$ 